\title{
Research and Analysis on DM6446 Da Vinci Platform-based Face Recognition System
}

\author{
Xiaoqing Luo, Rong Hu \\ Nanchang University College of Science and Technology, Nanchang 330029, China
}

\begin{abstract}
This paper mainly apply Da Vinci digital media processor DM6446 designed by TI Company as Central Processing Unit, which internally integrated dual-core, namely, ARM and DSP, it utilizes the high-speed data processing capacity of DSP for the operational processing of face recognition algorithm and performs contrast recognition and extraction of the face image characteristics, so as to provide a stable and effective operating control and interface support.
\end{abstract}

Keywords: Da Vinci platform, face recognition, DM6446

\section{Overview of face processing system}

Face tracking and checking are the research areas of computer vision and pattern recognition, as a very critical technology of face information processing technology, face detection technology has extensively spread and positively researched gradually in recent years, the applied face tracking systems in the past are often achieved through the computer system in large-scale integrated circuit, which also makes the increase in the overall cost of the system operation, while the reliability and stability can not be guaranteed. With the continuous progress of DPS technology and embedded technology, the applications of tracking system and face detection system have more reliable hardware protections. Identification certification is a basic activity in the daily life of the people, nowadays, we mostly rely on traditional markers such as passwords, keys and identification cards, and the shortcomings are obvious, biological recognition technology developed based on the biological characteristics of the people could radically reduce data theft and forgery with higher availability, security and reliability.

While the algorithm computation of face recognition has larger and higher complexity, various current face recognition systems are mainly developed with PC platform. With the optimizations and transplants made by various sectors to the face recognition system, the achievement of embedded face recognition system shows a greater significance gradually. This paper mainly apply Da Vinci digital media processor DM6446 designed by TI Company as Central Processing Unit, which internally integrated dual-core, namely, ARM and DSP, it utilizes the high-speed data processing capacity of DSP for the operational processing of face recognition algorithm and performs contrast recognition and extraction of the face image characteristics, so as to provide a stable and effective operating control and interface support. Based on the characteristics and resources of Da Vinci platform, this paper performs the optimi- 
zation and transplant of Gabor's characteristics extraction algorithm.

1. Hardware system structure. The central processing unit used in the system applies DM6446 Da Vinci platform, which is a high performance chip for image and video processing, and it internally integrates relevant programmable digital signal DSP core, video acceleration processor and ARM processor. Its function structure chart is shown in Figure 1:

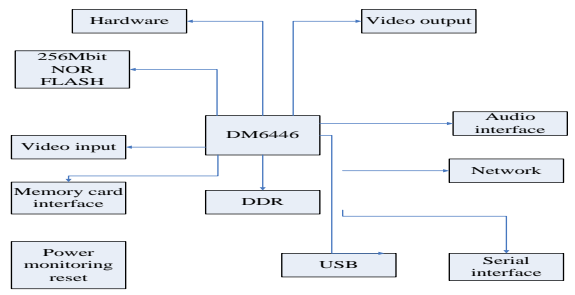

Fig. 1 DM6446 function structure chart

This type of DSP has a basic frequency of $594 \mathrm{MHz}$ and sixty four 32-bit generalpurpose registers and eight independent functional units, mainly including two multipliers and six arithmetic logic unit (ALU), it can perform four 16-bit additive and multiplicative computations or eight 8-bit additive and multiplicative computations in a monocyclic process by itself. The cache in this DSP chip totally includes $32 \mathrm{~KB}$ primary instruction cache and $80 \mathrm{~KB}$ primary data cache and $64 \mathrm{~KB}$ secondary instruction data cache, and these caches can be configured as cache or on-chip RAM.

2. Algorithm optimization and transplant of DSP. In multi-resolution image expression, the wavelet transform of Gabor is a relatively important way, the change of wavelet is a form in binary and directional of wavelet, and thus it has been widely applied in texture recognition. In 1985, Gabor function has been expanded into a 2-D form, and the 2-D filter constructed on this basis can not only get the minimum uncertainty of frequency domain and time, and it can approach to the signal received field model of mammal retinal neuron, where after determining the size of the window, the traditional transformation can not be changed in the entire process of the signal analysis, and can perform relevant analysis on fixed resolution. In order to optimize this deficiency, the people began to combine the changes of Gabor and wavelet theory, and proposed the resolution characteristics with wavelet transform and wavelet transform with the directionality and locality of Gabor function itself, so as to achieve better results in the process of edge detection and face image tracking. It's more complex with dimensionality to obtain the data characteristics of Gabor directly, where there are more redundancies and noises, LDA and PCA methods on traditional PC platform always apply the online ways, it trains the characteristics data in current database in the manner of real-time and can apply the on-line method on embedded platform to minimize the operating speed of the program to some extent and affect the effect of real-time. According to the above contents, this article mainly takes the offline method and selects a number of face databases with representative for relevant training, and it can convert the obtained data into fixed point data and store in the corresponding FLASH.

3. Da Vinci platform software design. DM6446 is a dual-core chip mainly including DSP core and ARM processor, the skeletons of these two cores itself are different, the communication between them may meet some problems, TI provides a software module, i.e., codec engine, which can be used to achieve the cooperative work between DSP and ARM systems, the software architecture of Da Vinci platform achieved by codec engine is shown in Figure 2.

The codec engine is an engine device between the signal processing layer and application layer, it plays a role as a bridge 
between DSP and ARM in the structure of dual-core software, the ARM application can call DSP algorithm by API provided by the codec engine, as shown in Figure 3, ARM calls API function VIDENC, the codec engine mainly locates at ARM side and opens the parameter A / B / C / and this information packet need to be called in the process by DSP side, the information is passed to both sides of DSP in the form of message queue, and the parameters of ARM side is converted into the corresponding parameters $\mathrm{x}, \mathrm{y}$, and $\mathrm{z}$ at DSP side, the system software architecture of ARM is shown in Figure 3.

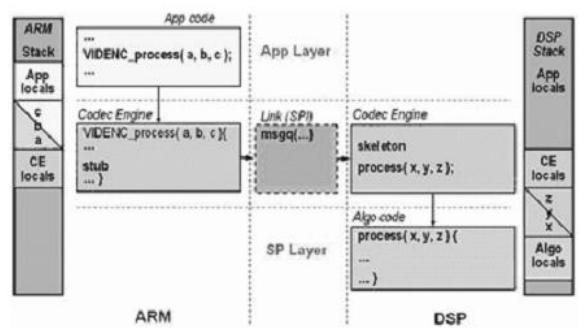

Fig. 2 Software architecture of Da Vinci platform

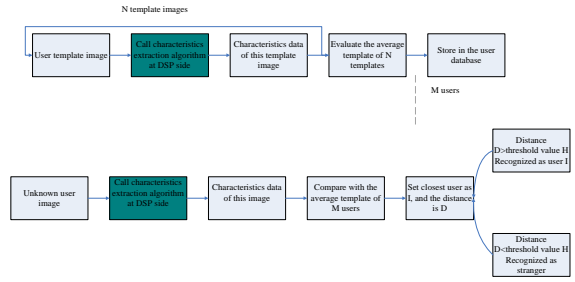

Fig. 3 System software architecture at ARM side

Before performing face recognition, ARM system needs to create a database about user characteristics for each customer, first of all, it needs to select $\mathrm{N}$ pictures to be used in the template creation of user characteristics, the pictures are mainly collected under the conditions such as the expression changes, attitude changes and light mounting pattern of the user, there will still be some differences, selecting the template image can enhance the effect of recognition, using the image with abundant elements can enable the recognition effect of system a good robustness to the facial expressions, gestures and light. Call the extracted module based on embedded Gabor characteristics of DSP and extract the characteristics of every template image and then draw the image characteristics data about this template, after completing all the characteristics extractions, it can draw the user's characteristics data template from the template characteristics data of $\mathrm{N}$ groups and store it into the database. After completing the creation of the user's template database, the system will automatically receive the image of unknown user and extract the characteristics, and it will make comparison between the obtained characteristics data and $\mathrm{N}$ group of user characteristics data template in the database, and calculate the distance between the relevant characteristics data, so as to obtain the most close user to the unknown image, and if the distance is D, and then judge the distance; if it's greater than the threshold set by the system, set the image recognition as user I, otherwise it can be judged to be a stranger.

\section{Experiment}

Select 611 staffs from the face database of CAS, and distribute three samples to each, and there are totally 1833 samples as training samples, select 966 from the database as the samples of closed set test, and then select 485 samples from which as the samples of open set test, and prepare all samples as experimental samples after face standardization. In the process of the open set test, it takes the processed pictures as experimental samples. In open set test, it applies the curve graph of Receiver Operating Characteristic to provide the false acceptance rate and false rejection rate values of different threshold 
values, the indicated valve selects the affect on FRR and FAR, and applies it to determine the performance of open set recognition, when the recognition rate shown in the results of closed set test reaches $98.5 \%$, the curve of open set test will be shown as below:

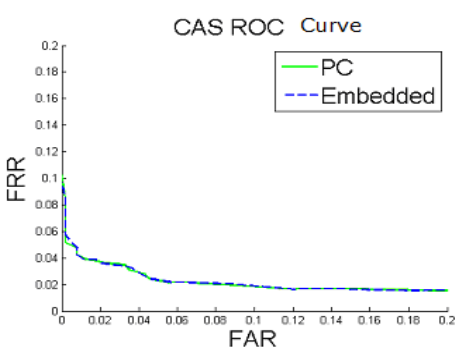

Fig. 4 ROC curves on CAS

We can learn from the experiments that the extraction of the embedded characteristics is relatively higher than the closed set recognition rate of recognition module by comparison, and its ROC curve is closer to PC system, while the performance of open set recognition is relatively good.

Due to the differences in Chinese and western cultures, the development of

\section{Conclusion}

This paper mainly makes related narratives on the development process and algorithm of Da Vinci platform-based face recognition system, from the point of view of the embedded system, it performs module extraction and improvement to Gabor characteristics on the traditional PC platform, puts forward characteristics extraction module for relevant offline fixed point, the experiments show that the system has better recognition performance, its experimental system has obtained commissioning and optimization currently, the system operation is also in relatively good condition. In the future work processes, it mainly includes posi- tioning function of characteristic points and adding in face detection function, so as to implement the automatic face recognition of the system and further improve the recognition performance of the system itself.

\section{Acknowledgement}

The paper is supported by the fund project of Youth Fund Project of Jiangxi Provincial Education Department. The Project name is: Da Vinci Platform-based Network Video Monitoring System (Number: GJJ12165).

\section{References}

[1] Belhumeur PN, Hespanha JP, Kriegman DJ. "Eigenfaces vs Fisherfaces: Recognition Using Class Specific Linear Projection." IEEE Transactions on Pattern Analysis and Machine Intelligence, 1997, 19 (7): 711-720.

[2] Bradski GR. "Computer Vision Face Tracking as a Component of a Perceptual User Interface". Proceedings of IEEE Workshop Applications of Computer Vision. Berlin, Germany. Princeton, NJ: IEEE, 1998, 10: 214219.

[3] Searcy J H, Bartlett J C. "Inversion and Processing of Component and Spatial-relational Information in Faces." Journal of Experimental Psychology: Human Perception and Performance, 1996, 22 (4): 904-915.

[4] Yang Wubing, Lu Xuping, Xu Jinhua. "Design of DSP and Face Recognition Technology-based Door Control System" . Electric Drive Automation, 2007, (02).

Li Wujun, Wang Chongjun, Zhang Wei, Chen Shifu. "Summary of Studies on Face Recognition". Pattern Recognition and Artificial Intelligence, 2006, (01). 